\title{
Monolithic RGB micro-light-emitting diodes fabricated with quantum dots embedded inside nanoporous GaN
}

Jie Song ${ }^{*}$, Jin-ho Kang ${ }^{2}$, and Jung Han²

${ }^{1}$ State Key Laboratory of Transient Optics and Photonics, Xi'an Institute of Optics and

Precision Mechanics, Chinese Academy of Sciences, Xi'an 710119, China

${ }^{2}$ Department of Electrical Engineering, Yale University, New Haven, Connecticut 06520,

United States

*Corresponding author: songjie@opt.ac.cn

Schematic drawing of of QD inkjet printing system

Figure S1 (a) shows the schematic illustration of an inkjet printing system in Taiwan National Chiao Tung University. Figure S1(b) shows the PL of the QDs used for the inkjet printing; the emission wavelength covers the spectrum from red $(630 \mathrm{~nm})$, green $(535 \mathrm{~nm})$, and blue (450 nm) light.

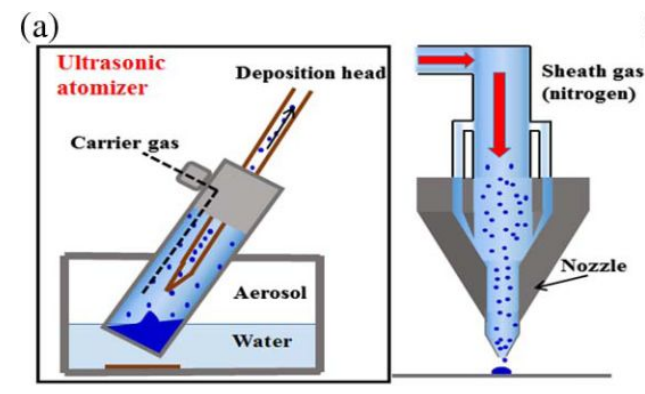

(b)

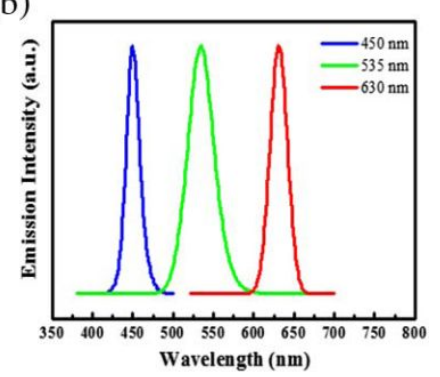


Figure S1. (a) Schematic illustration of the inkjet priting system. (b) The PL emission spectra of QDs with emission wavelenths of 450, 535, and $630 \mathrm{~nm}$.

\section{I-V characteristic of vertical micro-LEDs}

Figure S2 shows the I-V curve of vertical micro-LEDs shown in Figure 5.

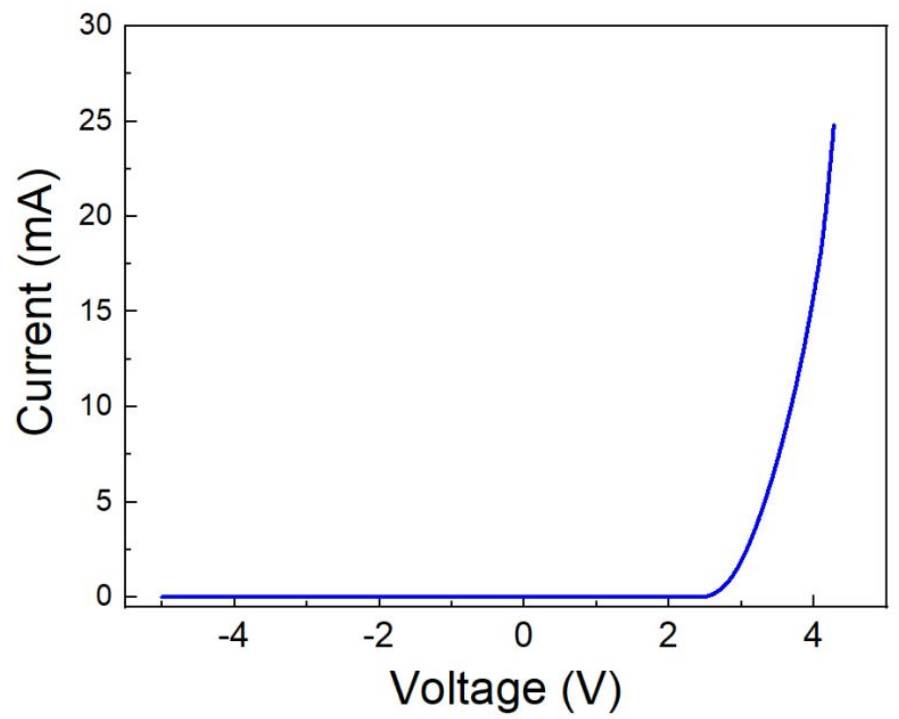

Figure S2. I-V curve of vertical micro-LEDs shown in Figure 5.

\section{Reliability of red QDs in nanoporous GaN under $85^{\circ} \mathrm{C}, 85 \%$ humidity}

Figure S3 shows the PL spetra of red QDs in nanoporous GaN before (black curve) and after (red curve) storing the QD sample in the ambient of $85^{\circ} \mathrm{C}, 85 \%$ humidity for 7 days. The Spectrum of red QDs sample show very little change in comparison after 7days storage in $85^{\circ} \mathrm{C}$, $85 \%$ humidity. 


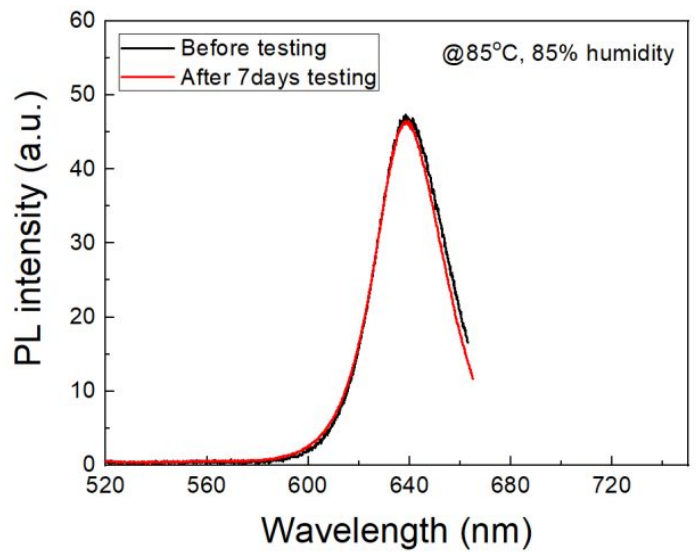

Figure S3. PL spetra of red QDs in nanoporous GaN before (black curve) and after (red curve) storing the QD sample in the ambient of $85^{\circ} \mathrm{C}, 85 \%$ humidity for 7 days. 